\title{
ALWAYS CONVERGENT METHODS FOR SOLVING NONLINEAR EQUATIONS
}

\author{
Aurél Galántai \\ Óbuda University \\ 1034 Budapest, Bécsi út 96/b, Hungary \\ galantai.aurel@nik. uni-obuda.hu
}

[Received: August 03, 2015, Accepted: August 24, 2015]

Dedicated to Professor Barna Szabó on the occasion of his eightieth birthday

\begin{abstract}
We develop always convergent methods for solving nonlinear equations of the form $f(x)=0\left(f: \mathbb{R}^{n} \rightarrow \mathbb{R}^{m}, x \in B=\times_{i=1}^{n}\left[a_{i}, b_{i}\right]\right)$ on continuous space curves that are lying in $B$. Under the only assumption that $f$ is continuous these methods have a kind of monotone convergence to the nearest zero on the given curve, if it exists, or the iterations leave the region in a finite number of steps. Depending on the selection of the curve these methods are always convergent in the previous sense. In the paper we also investigate the selection of curves and also provide numerical test results that indicate the feasibility of the suggested methods.
\end{abstract}

Mathematical Subject Classification: 65H05, 65H20

Keywords: Always convergent methods, nonlinear equations, space-filling functions, $\alpha$-dense curves

\section{INTRODUCTION}

Targonszky 1] investigated the following problem:

Let $f: \mathbb{C} \rightarrow \mathbb{C}$ be continuous in the closed domain

$$
S=\left\{z \in \mathbb{C}:|z| \leq K, \varphi_{0} \leq \arg z \leq \varphi_{1}\right\}
$$

and assume that $f(0) \neq 0$. Let

$$
\omega(\delta)=\sup _{\substack{z_{1}, z_{2} \in S \\\left|z_{1}-z_{2}\right| \leq \delta}}\left|f\left(z_{1}\right)-f\left(z_{2}\right)\right|
$$

be the modulus of continuity of $f$ and let $\rho:[0, \infty) \rightarrow[0, \infty)$ be a continuous and strictly monotone increasing function so that

$$
\rho(0)=0, \quad \rho(\delta) \geq \omega(\delta) \quad(\delta>0), \quad \lim _{\delta \rightarrow \infty} \rho(\delta)=\infty .
$$


Furthermore assume that $\gamma>0$ is a constant such that $\gamma K \leq 1$. Let $F:[0, \infty) \rightarrow$ $[0, \infty)$ be any continuous and strictly monotone decreasing function such that

$$
F(0)=1, \quad F(x) \geq \frac{1}{1+x} \quad(x>0) .
$$

Let

$$
\psi(z)=z F\left(\gamma \rho^{-1}(|f(z)|)\right) .
$$

Observe that for $z \neq 0, z=\psi(z)$ if and only if $f(z)=0$. For any $0 \neq z \in S$, $f(z) \neq 0$, define the iteration sequence

$$
\begin{aligned}
z_{0} & =z \\
z_{i+1} & =\psi\left(z_{i}\right), \quad i=0,1, \ldots .
\end{aligned}
$$

Theorem 1. ( [1] ). The iteration is always convergent in the following sense: (i) If the line segment $\{t z \in \mathbb{C}: 0<t \leq 1\}$ contains no zero of $f$, then $z_{i} \rightarrow 0$; (ii) If the line segment contains at least one zero of $f$, then $z_{i}$ tends to the zero that is nearest to $z$.

Theorem 2. ([1]). The line segment $\{t z \in \mathbb{C}: 0<t \leq 1\}$ contains no zero of $f$, if and only if the series $\sum_{n=0}^{\infty} z_{i}$ is convergent.

Denote by $\operatorname{Lip}_{M} \beta(0<\beta \leq 1)$ those functions for which

$$
\|f(x)-f(y)\| \leq M\|x-y\|^{\beta} \quad(x, y \in D \subset D(f)) .
$$

$\operatorname{Lip} \beta$ denotes the set of those functions that are $\operatorname{Lip}_{M} \beta$ for some constant $M \geq 0$.

If $f \in \operatorname{Lip}_{L} \beta(L>0)$, then $\omega(f ; \delta)=\rho(\delta)=L \delta^{\beta}$ can be chosen. Thus $\rho^{-1}(x)=$ $(x / L)^{1 / \beta}$ and the iteration function 1.1 takes the form

$$
\psi(z)=z F\left(\gamma\left(\frac{|f(z)|}{L}\right)^{\frac{1}{\beta}}\right) .
$$

It is interesting to note that Beauzamy $[2]$ investigated the direct paths from 0 to the zeros of polynomials $P$, that is the sets $\left\{P\left(t z_{j}\right): 0 \leq t \leq 1\right\}$, where $z_{j}$ is a zero of $P(z)$ (where $P$ is normalized so that $P(0)=1$ ). He showed that there is always a zero towards which the direct path declines near 0 , that is $\left|P\left(t z_{j}\right)\right|<|P(0)|$ if $t$ is small enough. However, starting with degree 5 , there are polynomials for which no direct path constantly remains below the altitude 1. Observe that Targonszky's method approaches the zero from above.

Also note that algorithm $1.2-1.3$ works with any function $f$ that is continuous on the compact set $S$. The weak point of the algorithm is, however, its behavior at zero. There is no indication if $f$ has no zero on the line segment, while the algorithm makes infinitely many iterations.

In this paper we extend Targonszky's method for solving nonlinear equations in more general environments. In Section 2 we analyze and generalize some of its properties. Then we develop some classes of iteration methods with better convergence 
behavior. Using the new results we suggest always convergent iteration schemes for solving nonlinear equations of the form

$$
f(x)=0 \quad\left(f: \mathbb{R}^{n} \rightarrow \mathbb{R}^{m}, x \in B=\times_{i=1}^{n}\left[a_{i}, b_{i}\right]\right),
$$

where $f$ is continuous on $B$. These methods work on continuous curves lying in $B$ and exhibit a kind of monotone convergence to the nearest zero on the given curve, if it exists, or the iterations leave the region in a finite number of steps. The convergence is assured under continuity and its rate is estimated in many cases. The suggested curves are space-filling curves and/or $\alpha$-dense curves.

Definition 3. Let $r:[0,1] \rightarrow[0,1]^{n}(n \geq 2)$ be a continuous mapping. The curve $x=r(t)(t \in[0,1])$ is space-filling if $r$ is surjective.

Given a space-filling curve $r:[0,1] \rightarrow[0,1]^{n}$ and the hyperrectangle (or box) $B=\times_{i=1}^{n}\left[a_{i}, b_{i}\right]$, the mapping

$$
h_{i}(t)=\left(b_{i}-a_{i}\right) r_{i}(t)+a_{i}, \quad i=1, \ldots, n
$$

clearly fills up the whole hyperrectangle $B$.

Space-filling curves are used in many areas $([3],[4],[5], 6])$. Their use in optimization was first suggested by Butz [7], 8], followed by Strongin and others (see, e.g. [9], 3], 6]). The use of Hilbert's space-filling functions for solving nonlinear systems was also suggested by Butz 10 and also later by Hlawka 11].

Definition 4. Let $I=[a, b] \subset \mathbb{R}$ be an interval and $B=\times_{i=1}^{n}\left[a_{i}, b_{i}\right] \subset \mathbb{R}^{n}$ be $a$ rectangle. The map $x: I \rightarrow B$ is an $\alpha$-dense curve, if for every $x \in B$, there exists a $t \in I$ such that $\|x(t)-x\| \leq \alpha$.

The concept and use of $\alpha$-dense curves in optimization was suggested and applied by Cherruault and Guillez (see, e.g. [12, [13 or [14]).

In Section 6 we analyze and select space-filling and $\alpha$-dense functions. In Section 8 we test some of the new methods on a set of test problems in order to see the feasibility of the suggested algorithms.

\section{An ANALYSis OF TARgONSZKY'S METHOD}

There are two key elements of Targonszky's method. One is the iteration formula, the other is a kind of Lipschitz property.

For the first element, we can reformulate the Targonszky method in the following way. Set $z_{i}=z t_{i}\left(t_{i} \in(0,1]\right)$. Then iteration function (1.1) can be replaced by

$$
\psi(t)=t F\left(\gamma \rho^{-1}(|f(z t)|)\right),
$$

where function $t F(y)$ has the following properties:

(i) $t F(y)$ is strictly monotone increasing in $t$ and strictly monotone decreasing in $y$;

(ii) For $t>0$ and $y>0, t F(y)<t$; 
(iii) For $t>0, t F(y)=t$ if and only if $y=0$.

In the next two sections we generalize this iteration method and also derive particular formulae.

The second element is that if $f$ has a zero $z^{*}=z t^{*} \in\{t z \in \mathbb{C}: 0<t \leq 1\}$, then $\gamma \rho^{-1}(|f(z t)|)$ satisfies a kind of Lipschitz property

$$
\gamma \rho^{-1}(|f(z t)|) \leq \frac{\left|t-t^{*}\right|}{t^{*}} .
$$

From the proof of Theorem 1 it seems that the latter property is the quintessential element of Targonszky's method. It can can be formulated more generally as follows.

Lemma 5. Assume that $f \in C[a, b], \omega(\delta)=\omega(f ; \delta)$ is its modulus of continuity, $\rho:[0, \infty) \rightarrow[0, \infty)$ is a strictly monotone increasing function such that $\rho(0)=0$, $\rho(x) \geq \omega(x)$ for $x \in[0, b-a]$ and $\lim _{x \rightarrow \infty} \rho(x)=\infty$. Assume that $f$ has a zero $\xi \in[a, b]$. Then

$$
\rho^{-1}(|f(x)|) \leq|x-\xi| \quad(x \in[a, b]) .
$$

Proof. Since $|f(x)|=|f(x)-f(\xi)| \leq \omega(|x-\xi|) \leq \rho(|x-\xi|)$ and $\rho^{-1}$ is also strictly monotone increasing we have $\rho^{-1}(|f(x)|) \leq|x-\xi|$.

If $f \in \operatorname{Lip}_{M} \beta$, then $\rho(\delta)=\omega(f ; \delta)=M \delta^{\beta}$ can be chosen. If $f$ is not Lipschitz $\beta$ for some $\beta(0<\beta \leq 1)$, then we can use the following result (for the proof, see Efimov [15]).

Theorem 6. (S.B. Stechkin). For each modulus of continuity $\omega(\delta), 0 \leq \delta \leq b-a$, there is a concave modulus of continuity $\omega_{1}(\delta)$ with the property

$$
\omega(\delta) \leq \omega_{1}(\delta) \leq 2 \omega(\delta), \quad 0 \leq \delta \leq b-a .
$$

Function $\omega_{1}$ is called the least concave majorant of $\omega$. It is easy to see that for $f \not \equiv 0$, either $\omega_{1}$ is strictly monotone on $[0, b-a]$ or it is strictly increasing on some interval $[0, c]$ and becomes constant on $[c, b-a]$.

In order to get a proper $\rho$ we can take any continuous and strictly monotone increasing function $g(x)$ that satisfies $g(0)=0, g(x) \geq x$, and $\lim _{x \rightarrow \infty} g(x)=\infty$. Then the function

$$
\rho(x)=\left\{\begin{array}{l}
g\left(x+\omega_{1}(x)\right), \quad 0 \leq x \leq b-a \\
g\left(x+\omega_{1}(b-a)\right), \quad x>b-a
\end{array}\right.
$$

will satisfy the requirements for $\rho$. For example, $g(x)=e^{x}-1$ is such a function.

Assume that $T \subset \mathbb{R}^{n}$ is a closed and bounded region and $f: \mathbb{R}^{n} \rightarrow \mathbb{R}^{m}$ is a continuous function on $T$. The modulus of continuity of $f$ is defined by

$$
\omega(\delta)=\omega(f ; \delta)=\sup _{\substack{x_{1}, x_{2} \in T \\\left\|x_{1}-x_{2}\right\| \leq \delta}}\left\|f\left(x_{1}\right)-f\left(x_{2}\right)\right\| \quad(0 \leq \delta \leq \operatorname{diam}(T)),
$$

where $\operatorname{diam}(T)$ is the diameter of the compact region $T$. It is clear that $\omega(\delta)$ is monotone decreasing. 
For other properties, Kolodii and Khil'debrand 16] proved the following results:

(i) $\omega(\delta)$ is continuous from the right;

(ii) $\omega(\delta)$ is continuous from the left if and only if $T$ satisfies the following condition A: for any $\delta>0$ and any points $x, y \in T, x \neq y$, there are points $x^{\prime}, y^{\prime} \in T$ such that $\left\|x^{\prime}-x\right\|<\delta,\left\|y^{\prime}-y\right\|<\delta$ and $\left\|x^{\prime}-y^{\prime}\right\|<\|x-y\|$;

(iii) $\omega(\delta)$ is continuous if and only if $T$ satisfies condition A;

(iv) $\omega$ is subadditive if and only if $T$ is convex.

Note that any region $T$ satisfying condition A is connected. It is also obvious that if $T$ is convex, then it satisfies condition A.

Lemma 7. Assume that $T \subset \mathbb{R}^{n}$ is a closed, bounded and convex region and $f: \mathbb{R}^{n} \rightarrow$ $\mathbb{R}^{m}$ is continuous on $T$. Let $\omega_{f}$ denote the modulus of continuity of $f$ on $T$. Let $\Gamma$ be any continuous curve that lays in $T$, that is $\Gamma=\{r(t): 0 \leq t \leq \tau\} \subset T$. Denote its modulus of continuity by $\omega_{r}$. Assume that $\rho_{f}, \rho_{r}:[0, \infty) \rightarrow[0, \infty)$ are continuous and strictly monotone increasing functions so that

$$
\rho_{f}(0)=0, \quad \rho_{f}(\delta) \geq \omega_{f}(\delta) \quad(\delta \in[0, \operatorname{diam}(T)]), \quad \lim _{\delta \rightarrow \infty} \rho_{f}(\delta)=\infty
$$

and

$$
\rho_{r}(0)=0, \quad \rho_{r}(\delta) \geq \omega_{r}(\delta) \quad(\delta \in[0, \tau]), \quad \lim _{\delta \rightarrow \infty} \rho_{r}(\delta)=\infty
$$

hold, respectively. If $f(r(t))$ has a zero $\xi$ on the curve $\Gamma$, that is $f(r(\xi))=0$ for some $\xi \in[0, \tau]$, then

$$
\rho_{r}^{-1}\left(\rho_{f}^{-1}(\|f(r(t))\|)\right) \leq|t-\xi| \quad(t \in[0, \tau]) .
$$

Proof. Since

$$
\|f(r(t))\|=\|f(r(t))-f(r(\xi))\| \leq \omega_{f}(\|r(t)-r(\xi)\|) \leq \rho_{f}(\|r(t)-r(\xi)\|),
$$

we have

$$
\rho_{f}^{-1}(\|f(r(t))\|) \leq\|r(t)-r(\xi)\| \leq \omega_{r}(|t-\xi|) \leq \rho_{r}(|t-\xi|)
$$

and

$$
\rho_{r}^{-1}\left(\rho_{f}^{-1}(\|f(r(t))\|)\right) \leq|t-\xi|
$$

Assume that $f \in \operatorname{Lip}_{L_{f}} \beta(0<\beta \leq 1)$. Then $\omega_{f}(\delta) \leq L_{f} \delta^{\beta}$ and we can select $\rho_{f}(\delta)=L_{f} \delta^{\beta}$ and $\rho_{f}^{-1}(\delta)=\left(\frac{\delta}{L_{f}}\right)^{1 / \beta}$. Similarly, if curve $\Gamma$ is $\operatorname{Lip}_{L_{\Gamma}} \mu(\mu \in(0,1])$, that is

$$
\|r(s)-r(t)\| \leq L_{\Gamma}|s-t|^{\mu} \quad(t, s \in[0, \tau]),
$$


then $\omega_{r}(\delta) \leq L_{\Gamma} \delta^{\mu}$ and so we can take $\rho_{r}(\delta)=L_{\Gamma} \delta^{\mu}$ and $\rho_{r}^{-1}(\delta)=\left(\frac{\delta}{L_{\Gamma}}\right)^{1 / \mu}$. Thus $\rho_{r}^{-1}\left(\rho_{f}^{-1}(\delta)\right)=\frac{1}{L_{\Gamma}^{\frac{1}{\mu}}}\left(\frac{\delta}{L_{f}}\right)^{\frac{1}{\mu \beta}}$ and in the case of a zero $\xi \in[0, \tau]$, we have the estimate

$$
\rho_{r}^{-1} \rho_{f}^{-1}(\|f(r(t))\|)=\frac{1}{L_{\Gamma}^{\frac{1}{\mu}}}\left(\frac{\|f(r(t))\|}{L_{f}}\right)^{\frac{1}{\mu \beta}} \leq|t-\xi| \quad(t \in[0, \tau]) .
$$

In Targonszky's case $r(t)=t$ for which $\mu=1, L_{\Gamma}=|z|$.

Corollary 8. Assume that both $f$ and $\Gamma$ are bi-Hölder, that is

$$
k_{f}\|x-y\|^{\beta} \leq\|f(x)-f(y)\| \leq L_{f}\|x-y\|^{\beta} \quad(x, y \in T)
$$

and

$$
k_{\Gamma}|s-t|^{\mu} \leq\|r(s)-r(t)\| \leq L_{\Gamma}|s-t|^{\mu} \quad(s, t \in[0, \tau]) .
$$

If $f(r(t))$ has a zero $\xi$ on the curve $\Gamma$, that is $f(r(\xi))=0$ for some $\xi \in[0, \tau]$, then

$$
\left(\frac{k_{\Gamma}}{L_{\Gamma}}\right)^{\frac{1}{\mu}}\left(\frac{k_{f}}{L_{f}}\right)^{\frac{1}{a \beta}}|t-\xi| \leq \rho_{r}^{-1} \rho_{f}^{-1}(\|f(r(t))\|) \leq|t-\xi|
$$

Proof. Since

$$
k_{f} k_{\Gamma}^{\beta}|t-\xi|^{\mu \beta} \leq\|f(r(t))\| \leq L_{f} L_{\Gamma}^{\beta}|t-\xi|^{\mu \beta}
$$

we have

$$
\rho_{r}^{-1}\left(\left[\frac{k_{f} k_{\Gamma}^{\beta}|t-\xi|^{\mu \beta}}{L_{f}}\right]^{1 / \beta}\right) \leq \rho_{r}^{-1} \rho_{f}^{-1}(\|f(r(t))\|) \leq \rho_{r}^{-1}\left(L_{\Gamma}|t-\xi|^{\mu}\right)=|t-\xi| .
$$

By definition

$$
\rho_{r}^{-1}\left(\left[\frac{k_{f} k_{\Gamma}^{\beta}|t-\xi|^{\mu \beta}}{L_{f}}\right]^{1 / \beta}\right)=\left(\frac{k_{\Gamma}}{L_{\Gamma}}\right)^{\frac{1}{\mu}}\left(\frac{k_{f}}{L_{f}}\right)^{\frac{1}{a \beta}}|t-\xi|,
$$

and so we have the two-sided bound

$$
\left(\frac{k_{\Gamma}}{L_{\Gamma}}\right)^{\frac{1}{\mu}}\left(\frac{k_{f}}{L_{f}}\right)^{\frac{1}{a \beta}}|t-\xi| \leq \rho_{r}^{-1} \rho_{f}^{-1}(\|f(r(t))\|) \leq|t-\xi| .
$$

If $\Gamma \subset \mathbb{R}$ is an interval, that is $r(t)=t$, then $k_{\Gamma}=L_{\Gamma}=1, \mu=1, \rho_{r}(t)=t$ and

$$
\left(\frac{k_{f}}{L_{f}}\right)^{\frac{1}{\beta}}|t-\xi| \leq \rho_{r}^{-1} \rho_{f}^{-1}(\|f(r(t))\|) \leq|t-\xi| .
$$




\section{A general Class of iteration methods}

We investigate iteration methods of the form

$$
x_{i+1}=F\left(x_{i}, \varphi\left(x_{i}\right)\right)
$$

for solving real equation $f(x)=0$ on the interval $[a, 1]([a, 1] \subset[0,1], f \in C[a, 1])$. The selection of interval $[0,1]$ is only for convenience. We assume that $F(x, y)=$ $x \Leftrightarrow y=0(x \geq a), F(x, y)>x$ or $F(x, y)<x$ for $y>0, \varphi(x) \geq 0$ and $\varphi(x)=$ $0 \Leftrightarrow f(x)=0$. We also assume that $y \geq 0$ and $y:=\varphi(x) \leq|x-\xi|$, if a zero $\xi$ of $f$ exists in the interval $[a, 1]$. Both $F$ and $\varphi$ are continuous, $F(x, y)$ is strictly monotone increasing in $x$ and strictly monotone in $y$. We separately investigate the case when $a=0$ and $F(0, y)=0$ may occur for some $y>0$.

Theorem 9. Assume that

(a) $F(x, y)$ is continuous in $[a, 1] \times[0, \infty)$;

(b) $x \geq a, F(x, y)=x \Leftrightarrow y=0$;

(c) $F(x, y)<x(x \in[a, 1], y>0)$;

(d) For $x>\xi(x, \xi \in[a, 1])$ and $0 \leq y \leq x-\xi, F(x, y) \geq \xi$.

(e) $F(x, y)$ is strictly monotone increasing in $x$, and strictly monotone decreasing in $y$;

Assume further that $\varphi(x) \geq 0, \varphi(\xi)=0 \Leftrightarrow f(\xi)=0$ and if a root $\xi \in[a, 1]$ exists, then $\varphi(x) \leq|x-\xi|$. Let $x_{0}=1$ and assume that $\varphi(1)>0$. Define

$$
x_{i+1}=F\left(x_{i}, \varphi\left(x_{i}\right)\right) \quad(i=0,1,2, \ldots) .
$$

Then $\left\{x_{i}\right\}$ is a strictly monotone decreasing sequence that converges to $\xi_{\max }$ if a root exists in $[a, 1]$. If no root exists, then the sequence $\left\{x_{i}\right\}$ leaves the interval $[a, 1]$ in a finite number of steps.

Proof. If $\varphi\left(x_{i}\right)>0$, then $x_{i+1}<x_{i}$ by (c). If $\varphi\left(x_{i}\right)=0$ then $x_{i+1}=x_{i}$ by (b). Assume that $x_{i}>\xi_{\max }$. Then $\varphi\left(x_{i}\right) \leq x_{i}-\xi_{\max }$ and $x_{i+1}=F\left(x_{i}, \varphi\left(x_{i}\right)\right) \geq$ $\xi_{\max }$ by $(\mathrm{d})$. Hence the sequence $\left\{x_{i}\right\}$ can not pass the zero $\xi_{\max }$. Since $\left\{x_{i}\right\}$ is a monotone decreasing sequence bounded from below, it has a limit point $x^{*}$ so that $\xi_{\max } \leq x^{*}=F\left(x^{*}, \varphi\left(x^{*}\right)\right)$. Hence $x^{*}=\xi_{\max }$. If there is no zero in $[a, 1]$, then $x-F(x, \varphi(x)) \geq m>0(x \in[a, 1])$ by $(\mathrm{c})$. Hence $x_{i+1} \leq x_{i}-m$ and for a large enough $i, x_{i}<a$.

Corollary 10. If for $x>\xi(x, \xi \in[a, 1])$ and $0 \leq y \leq x-\xi$,

$$
F(x, y) \geq \xi+\kappa_{2}(x-\xi) \quad\left(0<\kappa_{2}<1\right),
$$

and $\varphi(x)$ is such that $\varphi(x) \leq|x-\xi|(x \in[a, b])$, then the speed of convergence is at best linear.

Proof. Since $0 \leq \varphi\left(x_{i}\right) \leq x_{i}-\xi$, the assumption implies

$$
x_{i+1}-\xi_{\max }=F\left(x_{i}, \varphi\left(x_{i}\right)\right)-\xi_{\max } \geq \kappa_{2}\left(x_{i}-\xi_{\max }\right),
$$

which proves this. 
Corollary 11. If for $x>\xi(x, \xi \in[a, 1])$ and $0 \leq \theta(x-\xi) \leq y \leq x-\xi$,

$$
\xi+\kappa_{1}(x-\xi) \geq F(x, y)
$$

holds with constant $0<\kappa_{2}<\kappa_{1}<1$, and $\varphi(x)$ is such that $\theta|x-\xi| \leq \varphi(x) \leq|x-\xi|$ $(x \in[a, b])$, then the convergence speed is linear.

Proof. The assumption implies $\theta\left(x_{i}-\xi_{\max }\right) \leq \varphi\left(x_{i}\right) \leq x_{i}-\xi_{\max }$ and

$$
\kappa_{1}\left(x_{i}-\xi_{\max }\right) \geq x_{i+1}-\xi_{\max } \geq \kappa_{2}\left(x_{i}-\xi_{\max }\right) .
$$

If $a=0$ and $F(x, y)=x$ is possible for $y>0$ and $x=0$, then what we can prove is prove definitely less.

Theorem 12. Assume that

(a) $F(x, y)$ is continuous in $[0,1] \times[0, \infty)$;

(b) $x>0, F(x, y)=x \Leftrightarrow y=0$;

(c) $F(x, y)<x(x \in(0,1], y>0)$;

(d) For $x>\xi(x, \xi \in[0,1])$ and $0 \leq y \leq x-\xi, F(x, y) \geq \xi$.

(e) $F(x, y)$ is strictly monotone increasing in $x$, and strictly monotone decreasing in $y$;

Assume further that $\varphi(x) \geq 0, \varphi(\xi)=0 \Leftrightarrow f(\xi)=0$ and if a root $\xi \in[0,1]$ exists, then $\varphi(x) \leq|x-\xi|$. Let $x_{0}=1$ and assume that $\varphi(1)>0$. Define

$$
x_{i+1}=F\left(x_{i}, \varphi\left(x_{i}\right)\right) \quad(i=0,1,2, \ldots) .
$$

Then $\left\{x_{i}\right\}$ is a strictly monotone decreasing sequence that converges to $\xi_{\max }$ if a root exists in $[0,1]$. If no root exists, then the sequence $\left\{x_{i}\right\}$ either converges to 0 or leaves the interval $[0,1]$ in a finite number of steps.

Proof. If $\varphi\left(x_{i}\right)>0$, then $x_{i+1}<x_{i}$ by (c). If $\varphi\left(x_{i}\right)=0$ then $x_{i+1}=x_{i}$ by (b). Assume that $x_{i}>\xi_{\max }$. Then $\varphi\left(x_{i}\right) \leq x_{i}-\xi_{\max }$ and $x_{i+1}=F\left(x_{i}, \varphi\left(x_{i}\right)\right) \geq \xi_{\max }$ by (d). Hence the sequence $\left\{x_{i}\right\}$ can not pass the zero $\xi_{\max }$. Since $\left\{x_{i}\right\}$ is a monotone decreasing sequence bounded from below, it has a limit point $x^{*}$ so that $\xi_{\max } \leq x^{*}=$ $F\left(x^{*}, \varphi\left(x^{*}\right)\right)$. Hence $x^{*}=\xi_{\max }$. If there is no zero in $[0,1]$, then we have two possible cases: $x_{i} \geq 0$ for all $i$ or $x_{i_{0}}<0$ holds for some integer $i_{0}>0$. If $x_{i} \geq 0$ for all $i$ and we have no zero in $[0,1]$, then $x_{i} \rightarrow 0$ must hold.

Remark 13. The result is different from the previous one. The Targonszky case indicates that problems with $a=0$ if $F(0, y)=0$ may happen for $y>0$. The two corollaries of the previous theorem also hold.

Theorem 14. Assume that

(a1) $F(x, y)$ is continuous in $[a, 1] \times[0, \infty)$;

(b1) $x \geq a, F(x, y)=x \Leftrightarrow y=0$;

(c1) $F(x, y)>x(x \in[a, 1], y>0)$;

(d1) For $x<\xi(x, \xi \in[a, 1])$ and $0 \leq y \leq \xi-x, F(x, y) \leq \xi$;

(e1) $F(x, y)$ is strictly monotone increasing both in $x$ and $y$. 
Assume further that $\varphi(x) \geq 0, \varphi(\xi)=0 \Leftrightarrow f(\xi)=0$ and if a root $\xi \in[a, 1]$ exists, then $\varphi(x) \leq|x-\xi|$. Let $x_{0}=a$ and assume that $\varphi(a)>0$. Define

$$
x_{i+1}=F\left(x_{i}, \varphi\left(x_{i}\right)\right) \quad(i=0,1,2, \ldots) .
$$

Then $\left\{x_{i}\right\}$ is a strictly monotone increasing sequence that converges to $\xi_{\min }$ if a root exists in $[a, 1]$. If no root exists, then the sequence $\left\{x_{i}\right\}$ leaves the interval $[a, 1]$ in a finite number of steps.

Proof. If $\varphi\left(x_{i}\right)>0$, then $x_{i+1}>x_{i}$ by (c1). If $\varphi\left(x_{i}\right)=0$ then $x_{i+1}=x_{i}$ by (b1). Assume that $x_{i}>\xi_{\max }$. Then $\varphi\left(x_{i}\right) \leq \xi_{\min }-x_{i}$ and $x_{i+1}=F\left(x_{i}, \varphi\left(x_{i}\right)\right) \leq$ $\xi_{\text {min }}$ by $(\mathrm{d} 1)$. Hence the sequence $\left\{x_{i}\right\}$ cannot pass the zero $\xi_{\text {min }}$. Since $\left\{x_{i}\right\}$ is a monotone increasing sequence bounded from above, it has a limit point $x^{*}$ so that $\xi_{\min } \geq x^{*}=F\left(x^{*}, \varphi\left(x^{*}\right)\right)$. Hence $x^{*}=\xi_{\min }$. If there is no zero in $[a, 1]$, then $F(x, \varphi(x))-x \geq m>0(x \in[a, 1])$ by $(\mathrm{c} 1)$. Hence $x_{i+1} \geq x_{i}+m$ and for a large enough $i, x_{i}>1$.

Corollary 15. If for $x<\xi(x, \xi \in[a, 1])$ and $0 \leq y \leq \xi-x$,

$$
F(x, y) \leq \xi-\lambda_{2}(\xi-x) \quad\left(0<\lambda_{2}<1\right),
$$

and $\varphi(x)$ is such that $\varphi(x) \leq|x-\xi|(x \in[a, b])$, then the speed of convergence is at best linear.

Proof. The assumption implies

$$
\xi_{\text {min }}-x_{i+1}=\xi_{\min }-F\left(x_{i}, \varphi\left(x_{i}\right)\right) \geq \lambda_{2}\left(\xi_{\min }-x_{i}\right),
$$

which proves this.

Corollary 16. If for $x<\xi(x, \xi \in[a, 1])$ and $0 \leq \theta(\xi-x) \leq y \leq \xi-x$,

$$
\xi-\lambda_{1}(\xi-x) \leq F(x, y)
$$

holds with constants $0<\lambda_{2}<\lambda_{1}<1$, and $\varphi$ is such that $\theta|x-\xi| \leq \varphi(x) \leq|x-\xi|$ $(x \in[a, 1])$, then the convergence speed is linear.

Proof. The assumption implies $\theta\left(\xi_{\min }-x_{i}\right) \leq \varphi\left(x_{i}\right) \leq \xi_{\min }-x_{i}$ and

$$
\lambda_{2}\left(\xi_{\min }-x_{i}\right) \leq \xi_{\min }-x_{i+1} \leq \lambda_{1}\left(\xi_{\min }-x_{i}\right) .
$$

For certain cases $a=0$ is possible. For $F(x, y)=x(1+y)$ the iteration does not start from $x_{0}=0$. 


\section{Some iteration FunCtions}

Using requirements (a)-(e), (a1)-(e1) and various assumptions on the form of $F(x, y)$ such as

$$
\begin{gathered}
F(x, y)=g(x) h(y), \\
F(x, y)=g(x)+h(y),
\end{gathered}
$$

and

$$
F(x, y)=\frac{\alpha+\beta x+\gamma y}{a+b x+c y}
$$

we derived the iteration functions $(\mathrm{d}-1)-(\mathrm{d}-3)$, (i-1)-(i-3) given in the following tables. These tables also contain iteration functions (d-4), (i-4) that are direct generalizations of method 17] (see, also [18], [19]). It is assumed that function $U$ is strictly monotone increasing, and both $U$ and $U^{-1}$ are Lipschitz with $L_{U^{-1}}<1$ and $L_{U}>1$.

For the error constants $\kappa_{1}, \kappa_{2}, \lambda_{1}$ and $\lambda_{2}$ we have to assume the existence of a zero $\xi$.

\begin{tabular}{|l|l|l|l|}
\hline & monotone decreasing case & \multicolumn{1}{|c|}{$\kappa_{2}$} & $\kappa_{1}$ \\
\hline$(\mathrm{d}-1)$ & $F(x, y)=\frac{x}{1+y}$ & $(1-\xi) / 2 \geq 0$ & $1-\theta \xi \leq 1$ \\
\hline$(\mathrm{d}-2)$ & $F(x, y)=x-\frac{1}{P} y \quad(P \geq 1)$ & $1-\frac{1}{P} \geq 0$ & $1-\frac{\theta}{P}<1$ \\
\hline$(\mathrm{d}-3)$ & $\begin{array}{l}F(x, y)=\frac{p x+q y}{p+w y} \\
(p>0, W \geq 0, q<0, p \geq W-q)\end{array}$ & $\frac{p-w+q}{p+w} \geq 0$ & $\frac{p-\theta(w \xi-q)}{p}<1$ \\
\hline$(\mathrm{d}-4)$ & $F(x, y)=U^{-1}(U(x)-y)$ & $1-L_{U^{-1}}$ & $1-\frac{\theta}{L_{U}}$ \\
\hline
\end{tabular}

\begin{tabular}{|l|l|l|l|}
\hline & monotone increasing case & $\lambda_{2}$ & $\lambda_{1}$ \\
\hline (i-1) & $F(x, y)=x(1+y)$ & $1-x \geq 0$ & $1-\theta x \leq 1$ \\
\hline (i-2) & $F(x, y)=x+\frac{1}{P} y \quad(P \geq 1)$ & $1-\frac{1}{P} \geq 0$ & $1-\frac{\theta}{P}<1$ \\
\hline (i-3) & $F(x, y)=\frac{p x+q y}{p+w y} \quad(p \geq q>w \geq 0)$ & $\frac{p-(q-w \xi)}{p+w} \geq 0$ & $\frac{p-\theta(q-w \xi)}{p}<1$ \\
\hline (i-4) & $F(x, y)=U^{-1}(U(x)+y)$ & $1-L_{U^{-1}}$ & $1-\frac{\theta}{L_{U}}$ \\
\hline
\end{tabular}

Formulae (d-1) and (d-2) are special cases of (d-3). Case (d-1) is Targonszky's formula and $F(x, y)=x \Leftrightarrow y=0$ holds only for $x>0$.

Formula (i-2) is a special case of (i-3). For (i-1), $F(x, y)=x \Leftrightarrow y=0$ holds only for $x>0$.

If $U(x)=P x+q$, then $U^{-1}(x)=\frac{x-q}{P}$ and $U^{-1}(U(x) \pm y)=x \pm \frac{1}{P} y$. In this case formulae (d-2) and (i-2) are special cases of subclasses (d-4) and (i-4).

While the determination of error constants for cases (1)-(3) is rather straightforward, cases (d-4)-(i-4) require some argument. Here we exploit the strict monotonicity of $U$ and $U^{-1}$ as follows. For $x>\xi$,

$$
\begin{aligned}
U^{-1}(U(x)-y)-\xi & \geq \kappa_{2}(x-\xi) \Leftrightarrow U(x)-y \geq U\left(\xi+\kappa_{2}(x-\xi)\right) \Leftrightarrow \\
U(x)-U\left(\xi+\kappa_{2}(x-\xi)\right) & >\frac{1}{L_{U^{-1}}}\left(1-\kappa_{2}\right)(x-\xi) \geq x-\xi \geq y
\end{aligned}
$$


holds if $\frac{1}{L_{U^{-1}}}\left(1-\kappa_{2}\right) \geq 1$. This gives the error constant $\kappa_{2} \leq 1-L_{U^{-1}}$. Also we have

$$
\begin{aligned}
\kappa_{1}(x-\xi) & \geq F(x, y)-\xi \Leftrightarrow U\left(\kappa_{1}(x-\xi)+\xi\right) \geq U(x)-y \Leftrightarrow \\
U(x)-U\left(\kappa_{1}(x-\xi)+\xi\right) & \leq L_{U}\left(x-\xi-\kappa_{1}(x-\xi)\right) \\
& =L_{U}\left(1-\kappa_{1}\right)(x-\xi) \leq \theta(x-\xi) \leq y,
\end{aligned}
$$

that is if $L_{U}\left(1-\kappa_{1}\right) \leq \theta$, which leads to $\kappa_{1} \geq 1-\frac{\theta}{L_{U}}$.

Derivation of (i-4) error constants is similar. Note that in case $(\mathrm{d}-3) \kappa_{2}>0$, if $p>W-q$. Similarly in case (i-3) $\lambda_{2}>0$ if $p>q$.

\section{ITERATION METHODS FOR GENERAL EQUATIONS}

Here we study equations of the form

$$
f(x)=0 \quad\left(f: \mathbb{R}^{n} \rightarrow \mathbb{R}^{m}, x \in T=\times_{i=1}^{n}\left[a_{i}, b_{i}\right]\right),
$$

where $f$ is continuous on the (compact) rectangle/hyperinterval $T$, and each of the cases $n=m, n<m$ and $n>m$ is possible.

Assume that a continuous curve $\Gamma=\{r(t): 0 \leq t \leq 1\} \subset T$ is given. We seek for the solution of $f(x)=0$ on the curve $\Gamma$, that is the solution of equation

$$
f(r(t))=0 \quad(t \in[0,1]),
$$

which is equivalent to the real equation

$$
\|f(r(t))\|=0 \quad(t \in[0,1]) .
$$

Taking any iteration method $t_{i+1}=F\left(t_{i}, \varphi\left(t_{i}\right)\right)$ of Section 3 with

$$
\varphi(t)=\rho_{r}^{-1}\left(\rho_{f}^{-1}(\|f(r(t))\|)\right)
$$

we have an always convergent iteration method that either solves the equation on the curve $\Gamma$ or leaves $\Gamma$ in a finite number of steps.

One can easily reformulate Theorems 9, 12 and 14 and their respective Corollaries. We just do this with Theorem 9 .

Theorem 17. Assume that $f: \mathbb{R}^{n} \rightarrow \mathbb{R}^{m}$ is continuous on the rectangle $T=$ $\times_{i=1}^{n}\left[a_{i}, b_{i}\right]$ and $\Gamma=\{r(t): 0 \leq t \leq 1\} \subset T$ is a continuous curve. Let $\omega_{f}$ and $\omega_{r}$ be the modulus of continuity of $f$ on $T$ and $\Gamma$ on $[0,1]$, respectively. Assume that $\rho_{f}, \rho_{r}:[0, \infty) \rightarrow[0, \infty)$ are continuous and strictly monotone increasing functions so that

$$
\rho_{f}(0)=0, \quad \rho_{f}(\delta) \geq \omega_{f}(\delta) \quad(\delta \in[0, \operatorname{diam}(T)]), \quad \lim _{\delta \rightarrow \infty} \rho_{f}(\delta)=\infty
$$

and

$$
\rho_{r}(0)=0, \quad \rho_{r}(\delta) \geq \omega_{r}(\delta) \quad(\delta \in[0, \tau]), \quad \lim _{\delta \rightarrow \infty} \rho_{r}(\delta)=\infty
$$

hold, respectively. Furthermore assume that

(a) $F(x, y)$ is continuous in $[a, 1] \times[0, \infty)$;

(b) $x \geq a, F(x, y)=x \Leftrightarrow y=0$; 
(c) $F(x, y)<x(x \in[a, 1], y>0)$;

(d) For $x>\xi(x, \xi \in[a, 1])$ and $0 \leq y \leq x-\xi, F(x, y) \geq \xi$.

(e) $F(x, y)$ is strictly monotone increasing in $x$, and strictly monotone decreasing in $y$;

Define $\varphi(t)=\rho_{r}^{-1}\left(\rho_{f}^{-1}(\|f(r(t))\|)\right)(t \in[a, 1])$. Let $t_{0}=1$ and assume that $\varphi(1)>$ 0. Define

$$
t_{i+1}=F\left(t_{i}, \varphi\left(t_{i}\right)\right) \quad(i=0,1,2, \ldots) .
$$

Then $\left\{t_{i}\right\}$ is a strictly monotone decreasing sequence that converges to $\xi_{\max }$ if a root $\xi$ of $\|f(r(t))\|=0$ exists in $[a, 1]$. If no root exists, then the sequence $\left\{t_{i}\right\}$ leaves the interval $[a, 1]$ in a finite number of steps.

If $\Gamma$ is a space-filling curve, then we clearly have the always convergence property. Butz 10] suggested using the Hilbert curve, while Hlawka [11] suggested using the Schoenberg curve.

If the selected curve $\Gamma$ is not space-filling, the algorithm may fail to find a zero. However the space-filling functions used in practice are only approximations to the true ones, and do not have the space-filling property. Hence in practice we are forced to use approximate curves.

\section{Selection of Curves}

6.1. Space-filling curves. The first examples of space-filling functions were given by Peano in 2D and 3D and were followed by several other space-filling functions constructed by Hilbert, Lebesgue, Sierpinski, Schoenberg and many others (see, e.g. Singh 20], Sagan 21], Bader 22]). There are plenty of space-filling or Peano-type curves that are applied in a variety of fields (see, e.g. Strongin-Sergeyev [3, Zumbusch [4], Bebendorf [5], Sergeyev at al. [6]). The most often applied space-filling function is the Hilbert function (for definition, see, e.g. [21], Butz [7], 23], 3], [4], [6]).

Lemma 18. The Hilbert mapping $r_{H}:[0,1] \rightarrow[0,1]^{n}$ is space-filling, nowhere differentiable and $\operatorname{Lip}_{K} \mu$ with $K=2 \sqrt{n+3}$ and $\mu=1 / n$ :

$$
\left\|r_{H}(s)-r_{H}(t)\right\| \leq K|s-t|^{1 / n} \quad(s, t \in[0,1]) .
$$

For a proof, see, e.g. 4 (p. 96). Other $n$-dimensional space-filling functions that are Lip $\frac{1}{n}$ are the Peano and Sierpiński curves (see [4] p. 101).

Buckley 24 proved that there exist 2D space-filling curves $f$ that are Lip $\frac{1}{2}$, but no such curve is $\operatorname{Lip} \mu$ for $\mu>1 / 2$. Hence for $n=2$, the Lipschitz/Hölder-exponent of the Hilbert curve is the best possible. However the estimate of Lipschitz constant $K=2 \sqrt{5} \approx 4.4721$ is not the sharpest result. Bauman 25 proved that $K=2 \sqrt{5}$ can be replaced by $K=\sqrt{6}=2.4495$. For a more refined characterization of the smoothness properties of space-filling functions, see Jaffard-Nicolay [26].

Most of the space-filling functions are defined by a possibly recursive geometric process 21]. Any evaluation of the function at a point requires building up a good 
approximation to the whole function. We however need only to estimate the function at certain points.

From this point of view, Schoenberg's space filling-function [27], 28] is different, as it is defined by absolutely convergent series that can be evaluated at any point. Define function $p$ as

$$
p(t)= \begin{cases}0, & 0 \leq t \leq 1 / 3 \\ 3 t-1, & 1 / 3 \leq t \leq 2 / 3 \\ 1, & 2 / 3 \leq t \leq 4 / 3 \\ 5-3 t, & 4 / 3 \leq t \leq 5 / 3 \\ 0, & 5 / 3 \leq t \leq 2\end{cases}
$$

and extend it periodically by $p(t+2)=p(t)(t \in \mathbb{R})$. Note that $0 \leq p(t) \leq 1$ for any $t \in \mathbb{R}$. Also we have $|p(s)-p(t)| \leq 3|s-t|(s, t \in[0,2])$, which extends to $|p(s)-p(t)| \leq 3|s-t|(s, t \geq 0)$.

The two-dimensional space-filling curve of Schoenberg [27] is defined by

$$
r_{\mathrm{Sch}}(t)=[x(t), y(t)]^{T} \quad(t \in[0,1]),
$$

where

$$
x(t)=\frac{1}{2} \sum_{k=0}^{\infty} \frac{p\left(3^{2 k} t\right)}{2^{k}}, \quad y(t)=\frac{1}{2} \sum_{k=0}^{\infty} \frac{p\left(3^{2 k+1} t\right)}{2^{k}} .
$$

Steele 29 proved that Schoenberg's 2D curve is Lipschitz $\alpha$ with $\alpha=\frac{1}{2 \log _{2} 3} \approx$ 0.31546 (see also Jaffard-Nicolay [26]).

For general finite dimensional spaces Hlawka 11] extended Schoenberg's functions [27, 28]. The $n$-dimensional Schoenberg space-filling curve $r:[0,1] \rightarrow[0,1]^{n}$ is defined by

$$
r_{j}(t)=\sum_{k=0}^{\infty} \frac{1}{2^{k+1}} p\left(3^{n k+j-1} t\right) \quad(j=1, \ldots, n) .
$$

Using the proof of Steele [29] we estimate the Lipschitz exponent and also the Lipschitz constant.

Lemma 19. The $n$-dimensional Schoenberg curve is Lip $L_{L} \alpha$ with $\alpha=\frac{1}{\log _{2} 3^{n}}$ and Lipschitz constant $L \leq 3^{2 n} / \sqrt{2}$.

Proof. We can write

$$
\begin{aligned}
\left|r_{j}(s)-r_{j}(t)\right| & \leq \frac{1}{2} \sum_{k=0}^{\ell} \frac{\left|p\left(3^{n k+j-1} s\right)-p\left(3^{n k+j-1} t\right)\right|}{2^{k}}+\frac{1}{2} \sum_{k=\ell+1}^{\infty} \frac{2}{2^{k}} \\
& \leq \frac{3^{j}|s-t|}{2} \sum_{k=0}^{\ell}\left(\frac{3^{n}}{2}\right)^{k}+\frac{1}{2^{\ell+1}}
\end{aligned}
$$




$$
=\frac{3^{n+j}|s-t|}{2\left(3^{n}-2\right)}\left(\frac{3^{n}}{2}\right)^{\ell}+\frac{1}{2^{\ell+1}}
$$

Select $\ell$ so that

Then

$$
-\frac{\log _{2}|s-t|}{\log _{2} 3^{n}}<\ell \leq-\frac{\log _{2}|s-t|}{\log _{2} 3^{n}}+1
$$

$$
\left|r_{j}(s)-r_{j}(t)\right| \leq \frac{1}{2}\left(\frac{3^{2 n+j}}{3^{n}-2}+1\right)|s-t|^{\frac{1}{\log _{2} 3^{n}}}
$$

and

$$
\|r(s)-r(t)\|_{2}^{2} \leq\left[\frac{1}{4} \sum_{j=1}^{n}\left(\frac{3^{2 n+j}}{3^{n}-2}+1\right)^{2}\right]|s-t|^{\frac{2}{\log _{2} 3^{n}}} .
$$

A simple calculation yields the estimates for $n \geq 2$ and $1 \leq j \leq n$,

$$
\frac{3^{2 n+j}}{3^{n}-2}+1 \leq \frac{4}{3} 3^{n+j}
$$

and

$$
\frac{1}{4} \sum_{j=1}^{n}\left(\frac{3^{2 n+j}}{3^{n}-2}+1\right)^{2} \leq \frac{1}{2} 3^{2 n}\left(3^{2 n}-1\right) .
$$

Hence we obtain the estimate

$$
\|r(s)-r(t)\|_{2} \leq \frac{3^{2 n}}{\sqrt{2}}|s-t|^{\frac{1}{\log _{2} 3^{n}}} .
$$

A direct substitution into formula 6.6 gives a somewhat better result. For example for $n=2$ and $n=3$, we obtain $L=55.54\left(\operatorname{vs~} 3^{4} / \sqrt{2}=57.276\right.$ ) and $L=417.95\left(\right.$ vs. $\left.3^{6} / \sqrt{2}\right)=515.48$, respectively. Note however that

$$
\lim _{n \rightarrow \infty} \frac{\frac{1}{4} \sum_{j=1}^{n}\left(\frac{3^{2 n+j}}{3^{n}-2}+1\right)^{2}}{\frac{1}{2} 3^{4 n}}=\frac{9}{16} \text {. }
$$

Note that the $n$-dimensional Lebesgue space-filling curve is also $\operatorname{Lip} \frac{1}{\log _{2} 3^{n}}$ (see 4 p.108). Both curves are based on the Cantor set and closely related (see, e.g. 27], [4, [26, 30]).

6.2. Alpha-dense curves. The idea of $\alpha$-dense curves comes from the Alienor method of optimization and it is due to Cherruault and Guillez (see, e.g. [12, [13]). The theory and application of $\alpha$-dense curves is summarized in the monograph by Cherruault and Mora [14].

Definition 20. Let $I=[a, b] \subset \mathbb{R}$ be an interval and $B=\times_{i=1}^{n}\left[a_{i}, b_{i}\right] \subset \mathbb{R}^{n}$ be $a$ rectangle. The map $x: I \rightarrow B$ is an $\alpha$-dense curve, if for every $x \in B$, there exists a $t \in I$ such that $\|x(t)-x\| \leq \alpha$. 
There are many $\alpha$-dense curves (see, e.g. [14]). Particularly we mention two curves:

$$
x_{i}(t)=\frac{1}{2}\left(1-\cos \left(\omega_{i} 2 \pi t\right)\right), \quad i=1, \ldots, n
$$

by Cherruault and

$$
\begin{aligned}
& x_{1}(t)=t, \\
& x_{i}(t)=\frac{1}{2}\left(1-\cos \left(\sigma^{i-1} 2 \pi t\right)\right), \quad i=2, \ldots, n
\end{aligned}
$$

by Mora.

Note that approximations of space-filling curves are also $\alpha$-dense curves for some $\alpha$. For 2D, the $n$th approximating polygon of the Hilbert curve is $\alpha$-dense with $\alpha \leq \sqrt{2} / 2^{2 n}$, while for $3 \mathrm{D}, \alpha \leq \sqrt{3} / 2^{3 n}$ (see, e.g. Sagan 21]).

Mora [31] gave a characterization of the connection between space-filling curves and $\alpha$-dense curves.

The $\alpha$-dense curves are not space-filling functions. Hence for any small $\alpha>0$, the solver can fail. This may happen, however, with the approximate space-filling functions as well.

However it is easy to evaluate/compute $\alpha$-dense curves and they may be smooth enough. It is a disadvantage that the known constructions are such that their Lipschitz constant grows to infinity, while $\alpha \rightarrow 0$.

We use the following class of $\alpha$-dense curves.

Assume that $f_{i}:[0,1] \rightarrow[0,1](i=1, \ldots, n)$ are continuous functions such that for each $i=1,2, \ldots, n$, Range $\left(f_{i}\right)=[0,1]$ and $f_{i}$ is periodically extended to $\mathbb{R}$ with period 1. Also assume that there exist constants $L_{i}>0$ and $0<\beta_{i} \leq 1$ such that

$$
\left|f_{i}(x)-f_{i}(y)\right| \leq L_{i}|x-y|^{\beta_{i}} \quad(x, y \in[0,1])
$$

hold for $i=1,2, \ldots, n$. Define the curve $x:[0,1] \rightarrow[0,1]^{n}$ by

$$
x_{i}(t)=f_{i}\left(\omega_{i} t\right), \quad i=1, \ldots, n,
$$

where $\omega_{i}$ 's are positive integers such that $\omega_{i+1}=\sigma_{i} \omega_{i}$ with $\sigma_{i} \in \mathbb{N}$ and $\sigma_{i} \geq 1$.

It is clear that $x_{i}(t)$ is periodic with period $T_{i}=1 / \omega_{i}$. Note that $T_{i}=\sigma_{i} T_{i+1}$.

Lemma 21. The density of curve 6.11) is

$$
\alpha \leq\left(\sum_{i=1}^{n} \frac{L_{i}^{2}}{\left(\sigma_{i}^{\beta_{i}}\right)^{2}}\right)^{1 / 2}
$$

Proof. Choose any point $a \in[0,1]^{n}$. We estimate the distance between $a$ and the curve $x(t)$. There exists $t_{1} \in\left[0, \frac{1}{\omega_{1}}\right]$ such that $x_{1}\left(t_{1}\right)=a_{1}$. There is a unique integer 
$0 \leq k_{1} \leq \sigma_{1}-1$ such that $t_{1} \in I_{1}=\left[\frac{k_{1}}{\omega_{2}}, \frac{1+k_{1}}{\omega_{2}}\right]$. Note that for any $t \in I_{1}$,

$$
\left|x_{1}(t)-a_{1}\right|=\left|f_{1}\left(\omega_{1} t\right)-f_{1}\left(\omega_{1} t_{1}\right)\right| \leq L_{1}\left(\omega_{1}\left|t-t_{1}\right|\right)^{\beta_{1}} \leq \frac{L_{1}}{\sigma_{1}^{\beta_{1}}} .
$$

There exists $t_{2} \in\left[0, \frac{1}{\omega_{2}}\right]$ such that $x_{2}\left(t_{2}\right)=a_{2}$. There is a unique integer $0 \leq k_{2} \leq$ $\sigma_{2}-1$ such that $t_{2} \in\left[\frac{k_{2}}{\omega_{3}}, \frac{1+k_{2}}{\omega_{3}}\right]$. For any $t \in\left[\frac{k_{2}}{\omega_{3}}, \frac{1+k_{2}}{\omega_{3}}\right]$,

$$
\left|x_{2}(t)-a_{2}\right|=\left|f_{2}\left(\omega_{2} t\right)-f_{2}\left(\omega_{2} t_{2}\right)\right| \leq L_{2}\left(\omega_{2}\left|t-t_{2}\right|\right)^{\beta_{2}} \leq \frac{L_{2}}{\sigma_{2}^{\beta_{2}}} .
$$

Define $I_{2}=\left[\frac{k_{1}}{\omega_{2}}+\frac{k_{2}}{\omega_{3}}, \frac{k_{1}}{\omega_{2}}+\frac{1+k_{2}}{\omega_{3}}\right]$. Since $\frac{1+k_{2}}{\omega_{3}} \leq \frac{1}{\omega_{2}}, I_{2} \subset I_{1}$. The periodicity assumption implies that for $t \in I_{2}$,

$$
\left|x_{2}(t)-a_{2}\right| \leq L_{2}\left(\omega_{2}\left|t-t_{2}\right|\right)^{\beta_{2}} \leq \frac{L_{2}}{\sigma_{2}^{\beta_{2}}}
$$

also holds. Assume that for $j \geq 2$, we have the interval

$$
I_{j}=\left[\sum_{i=1}^{j} \frac{k_{i}}{\omega_{i+1}}, \frac{1}{\omega_{j+1}}+\sum_{i=1}^{j} \frac{k_{i}}{\omega_{i+1}}\right] \subset I_{j-1}
$$

such that for $t \in I_{j},\left|x_{j}(t)-a_{j}\right| \leq L_{j} / \sigma_{j}^{\beta_{j}}$ holds. There exists $t_{j+1} \in\left[0, \frac{1}{\omega_{j+1}}\right]$ such that $x_{j+1}\left(t_{j+1}\right)=a_{j+1}$. There is a unique integer $0 \leq k_{j+1} \leq \sigma_{j+1}-1$ such that $t_{j+1} \in\left[\frac{k_{j+1}}{\omega_{j+2}}, \frac{1+k_{j+1}}{\omega_{j+2}}\right]$. For any $t \in\left[\frac{k_{j+1}}{\omega_{j+2}}, \frac{1+k_{j+1}}{\omega_{j+2}}\right]$,

$$
\left|x_{j+1}(t)-a_{j+1}\right| \leq L_{j+1}\left(\omega_{j+1}\left|t-t_{j+1}\right|\right)^{\beta_{j+1}} \leq \frac{L_{j+1}}{\sigma_{j+1}^{\beta_{j+1}}} .
$$

Define

$$
I_{j+1}=\left[\sum_{i=1}^{j+1} \frac{k_{i}}{\omega_{i+1}}, \frac{1}{\omega_{j+2}}+\sum_{i=1}^{j+1} \frac{k_{i}}{\omega_{i+1}}\right] .
$$

Since $\frac{1+k_{j+1}}{\omega_{j+2}} \leq \frac{1}{\omega_{j+1}}, I_{j+1} \subset I_{j}$. By the periodicity of $x_{j+1}(t)$, for $t \in I_{j+1}$,

$$
\left|x_{j+1}(t)-a_{j+1}\right| \leq L_{j+1}\left(\omega_{j+1}\left|t-t_{j+1}\right|\right)^{\beta_{j+1}} \leq L_{j+1} / \sigma_{j+1}^{\beta_{j+1}}
$$

Thus we have a sequence of intervals $I_{n} \subset I_{n-1} \subset \cdots \subset I_{1}$ such that for any $t \in I_{n}$,

$$
\left|x_{j}(t)-a_{j}\right| \leq \frac{L_{j}}{\sigma_{j}^{\beta_{j}}} \quad(j=1,2, \ldots, n) .
$$

Hence the density of $x(t)$ is estimated by

$$
\|x(t)-a\|_{2}^{2} \leq \sum_{i=1}^{n} \frac{L_{i}^{2}}{\left(\sigma_{i}^{\beta_{i}}\right)^{2}} .
$$


Remark 22. Since $\sigma_{i}$ is specified by $\omega_{i+1}=\sigma_{i} \omega_{i}$ for $i=1, \ldots, n-1, \sigma_{n} \geq 1$ is our choice. If $L=L_{j}, \sigma_{i}=\sigma>1$ and $\beta_{i}=1(i=1, \ldots, n-1)$, then we can select $\sigma_{n}=\sigma$ and so the density estimate becomes

$$
\|x(t)-a\|_{2} \leq \frac{\sqrt{n} L}{\sigma} .
$$

If $f_{1}(t)=t, \omega_{1}=1$ and $L=L_{j}, \sigma_{i}=\sigma>1, \beta_{i}=1(i=2, \ldots, n)$, then

$$
\|x(t)-a\|_{2} \leq \frac{\sqrt{1+(n-1) L^{2}}}{\sigma} .
$$

Remark 23. It is clear from the proof that for $f_{1}(t)=t$, we do not need its periodic extension to $\mathbb{R}$. It also follows that for $t_{1} \neq t_{2}, x\left(t_{1}\right) \neq x\left(t_{2}\right)$, unlike in the case of space-filling functions.

If $f_{i}$ is periodically extended to $\mathbb{R}$, then its Lipschitz constant may change.

Lemma 24. If $f_{i}$ is continuously and periodically extended to $\mathbb{R}$, then

$$
\left|f_{i}(s)-f_{i}(t)\right| \leq 2^{1-\beta_{i}} L_{i}|s-t|^{\beta_{i}} \quad(s, t \geq 0) .
$$

Proof. Assume that $\beta_{i}=1$. If $s, t \in[k, k+1]$ ( $k \geq 0$ integer), then

$$
\left|f_{i}(s)-f_{i}(t)\right|=\left|f_{i}(s-k)-f_{i}(t-k)\right| \leq L_{i}|s-t|,
$$

which clearly extends to $\mathbb{R}$. Assume that $\beta_{i}<1$. If $s, t \in[k, k+1](k \geq 0$ integer $)$, then

$$
\left|f_{i}(s)-f_{i}(t)\right|=\left|f_{i}(s-k)-f_{i}(t-k)\right| \leq L_{i}|s-t|^{\beta_{i}} .
$$

If, say $s>t$ and $s \in[k, k+1], t \in[j, j+1]$ and $k \geq j+1$, then by the periodicity and the continuity

$$
\left|f_{i}(s)-f_{i}(t)\right|=\left|f_{i}(s)-f_{i}(k)+f_{i}(j+1)-f_{i}(t)\right| \leq L_{i}\left(|s-k|^{\beta_{i}}+|j+1-t|^{\beta_{i}}\right) .
$$

Since $x^{\beta_{i}}$ is concave, the Jensen inequality implies that

$$
|s-k|^{\beta_{i}}+|j+1-t|^{\beta_{i}} \leq 2\left(\frac{s-k+j+1-t}{2}\right)^{\beta_{i}} \leq 2\left(\frac{s-t}{2}\right)^{\beta_{i}}
$$

and

$$
\left|f_{i}(s)-f_{i}(t)\right| \leq 2^{1-\beta_{i}} L_{i}|s-t|^{\beta_{i}}
$$

Corollary 25. Curve 6.11) satisfies the Lipschitz condition

$$
\|x(t)-x(s)\|_{2} \leq\left[\sum_{i=1}^{n}\left(2^{1-\beta_{i}} L_{i} \omega_{i}^{\beta_{i}}|s-t|^{\beta_{i}}\right)^{2}\right]^{1 / 2} \quad(s, t \in[0,1]) .
$$

Particularly, if $L=L_{j}, \sigma_{i}=\sigma>1$ and $\beta_{i}=1(i=1, \ldots, n)$, then

$$
\|x(t)-x(s)\|_{2} \leq \omega_{1} L\left(\frac{\sigma^{2 n}-1}{\sigma^{2}-1}\right)^{1 / 2}|s-t| \quad(s, t \in[0,1]) .
$$


If $f_{1}(t)=t, \omega_{1}=1$ and $L=L_{j}, \sigma_{i}=\sigma>1, \beta_{i}=1(i=2, \ldots, n)$, then

$$
\|x(t)-x(s)\|_{2} \leq\left(1+L^{2} \frac{\sigma^{2 n}-\sigma^{2}}{\sigma^{2}-1}\right)^{1 / 2}|s-t| \quad(s, t \in[0,1]) .
$$

\section{COMPARISON With KNOWN METHODS}

The use of space filling functions for solving nonlinear equations first appeared in Butz [10] and later in Hlawka [11. In the context of the theory of uniform distributions Hlawka suggested a quadrature related method that uses Schoenberg's space-filling curve and is different from the present approach.

Butz [10] investigated the following problem

$$
f(x)=0 \quad\left(f: X \subset[0,1]^{n} \rightarrow \mathbb{R}^{N}\right),
$$

where $X$ is nonempty and closed. Let $h:[0,1] \rightarrow[0,1]^{n}$ be a space-filling curve that satisfies the Lipschitz condition

$$
\|h(t)-h(t+\Delta)\| \leq B(\Delta t, t) \leq M \Delta^{1 / n} \quad(t, t+\Delta t \in[0,1], \Delta t \geq 0),
$$

where $B$ is a nondecreasing function of $\Delta t$, and $B=0$ if and only if $\Delta t=0$.

Define

and $\bar{x}(X)=h(\bar{t}(X))$.

$$
\bar{t}(X)=\min \{t: t \in[0,1], h(t) \in X\}
$$

Theorem 26. (Butz [10]) Assume that $\omega(x)$ is some function and $\gamma(x)$ is some continuous function such that for all $x \in[0,1]^{n}$,

$$
\omega(x) \geq \gamma(x) \geq 0
$$

with strict inequality on the right if $x \notin X$ and, if $X$ is not empty,

$$
\omega(x) \leq\left\|x-x^{\prime}\right\|
$$

for every $x^{\prime} \in X$. Let $\left\{t^{i}, x^{i}\right\}$ be a sequence, where $t^{0}=0$ and $x^{i}=h\left(t^{i}\right)$ such that

$$
t^{i+1}=\min \left\{1, t^{i}+\Delta t^{i}\right\}
$$

where $\Delta t^{i} \geq 0$ is such that

$$
\omega\left(x^{i}\right) \geq B\left(\Delta t^{i}, t^{i}\right) \geq \min \left\{\kappa, \frac{1}{2} \omega\left(x^{i}\right)\right\},
$$

where $\kappa>0$ is some constant, with strict inequality on the left if $\omega\left(x^{i}\right)>0\left(x^{i} \notin X\right)$. Then if $X$ is not empty, $t^{i} \rightarrow \bar{t}(X)$ and $x^{i} \rightarrow \bar{x}(X)$ with $t^{i+1}>t^{i}$ for all $i$ (unless $h(0) \in X)$. If $X$ is empty, $t^{i}=1$ for finite $i$ with $\omega(h(1))>0$.

Butz applies this result and Hilbert's space-filling function to solve equation

$$
f(x)=0 \quad\left(f:[0,1]^{n} \rightarrow \mathbb{R}^{N}\right),
$$

when $X$ is the solution set, and it is assumed that for any solution $x^{*} \in X$,

$$
\left|f_{k}(x)\right| \leq K_{k}\left\|x-x^{*}\right\| \quad\left(x \in[0,1]^{n}, k=1, \ldots, N\right)
$$


holds with positive constants $K_{k}$. Using

$$
\omega(x)=\max _{1 \leq k \leq N} \frac{\left|f_{k}(x)\right|}{K_{k}} \quad\left(\leq\left\|x-x^{*}\right\|\right)
$$

his algorithm $(7.6)$ takes the form

$$
t^{i+1}=t^{i}+\left(\frac{\omega\left(x^{i}\right)}{M}\right)^{n}
$$

(for this see [10, p. 379).

Since $f_{k}(x)=0 \Leftrightarrow f_{k}(x) / K_{k}=0$, we can assume that equation $f(x)=0$ is such that every $K_{k}=1$. Then $\omega(x)=\max _{k}\left|f_{k}(x)\right|=\|f(x)\|_{\infty}$. Here $\rho_{r}(\delta)=M \delta^{1 / n}$ and $\rho_{f}(\delta)=\delta, \rho_{r}^{-1}(\delta)=\left(\frac{\delta}{M}\right)^{n}$ and $\rho_{f}^{-1}(\delta)=\delta$. Hence

$$
\rho_{r}^{1}\left(\rho_{f}^{-1}(\delta)\right)=\rho_{r}^{-1}(\delta)=\left(\frac{\delta}{M}\right)^{n}
$$

and

$$
\rho_{r}^{1}\left(\rho_{f}^{-1}(\|f(r(t))\|)\right)=\left(\frac{\|f(r(t))\|}{M}\right)^{n}
$$

Hence formula 7.10 clearly corresponds to iteration function (i-2) with $P=1$.

\section{NumericAl EXPERIMENTS}

The purpose of testing is only to get some view about the feasibility and behavior of the suggested algorithms. It is clear that space-filling curves have no finite arclength and so the computation time to get the first zero on the curve (if it exists) can be arbitrarily high. The smooth $\alpha$-dense curves have finite arclength that increases to infinity when $\alpha \rightarrow 0$.

8.1. The tested algorithms and curves. 1. Targonszky's extended formula ((d1))

$$
F(x, y)=\frac{x}{1+y}
$$

2. Formula (d-2) with $P=1$ :

$$
F(x, y)=x-y
$$

This formula corresponds to formula 7.10 of Butz.

3. Formula (d-3) with fixed parameters

$$
F(x, y)=\frac{x-0.5 y}{1+0.5 y} .
$$

4. Formula (d-4) with $U(x)=(x+2)^{2}$ :

$$
F(x, y)=\sqrt{(x+2)^{2}-y}-2 .
$$


The tested curves were the Hilbert and Schoenberg space-filling-curves and the following $\alpha$-dense curves: Cherruault curve with $\omega_{i}=\sigma^{i}$, Mora's curve, and three other curves of the form

$$
\begin{aligned}
& x_{1}(t)=t \\
& x_{i}(t)=f\left(\sigma^{i-1} t\right), \quad i=2, \ldots, n,
\end{aligned}
$$

with $f(x)=p(2 x)$ (Schoenberg's $p(x)$, identified as Schoenberg- $\alpha$ ), $f(x)=1$ $|2 x-1|$ (identified as ADC1) and

$$
f(x)= \begin{cases}\frac{3}{2} x+\frac{1}{2}, & 0 \leq x \leq 1 / 3 \\ 2-3 x, & 1 / 3 \leq x \leq 2 / 3 \\ \frac{3}{2} x-1, & 2 / 3 \leq x \leq 1\end{cases}
$$

(identified as ADC2).

For the computation of the 2D Hilbert curve we used the algorithm on page 52 of Bader 22] with depth $=50$, that computes the points of the curve with an error proportional to $2^{-50}=8.8818 \times 10^{-16}$. For $3 \mathrm{D}$, we used a recursively generated approximate Hilbert curve with 2097152 points and density $\alpha \approx 0.0078$. The error of the used Schoenberg curve approximation is also proportional to $2^{-50}=8.8818 \times$ $10^{-16}$.

The problem of exit or termination condition is well-known both from practical and theoretical points of view (see, e.g. Rice [32], Delahaye 33]). We set generally the condition

$$
\left\|f\left(r\left(t_{i}\right)\right)\right\| \leq t o l \vee i=i t \max
$$

which is not the most sophisticated exit condition.

It is reasonable to have the practical lower bound $\varphi\left(t_{i}\right) \geq \varepsilon_{\text {machine }}$ on the iterates $t_{i}$. For $f \in \operatorname{Lip}_{L_{f}} \beta$ and $r \in \operatorname{Lip}_{L_{\Gamma}} \mu$, this holds if and only if $\left\|f\left(r\left(t_{i}\right)\right)\right\| \geq$ $L_{f} L_{\Gamma}^{\beta} \varepsilon_{\text {machine }}^{\mu \beta}$. Hence the tol parameter has the lower bound tol $\geq L_{f} L_{\Gamma}^{\beta} \varepsilon_{\text {machine }}^{\mu \beta}$. Since each of the tested problems has $\beta=1$, the lower bound changes to tol $\geq$ $L_{f} L_{\Gamma} \varepsilon_{\text {machine }}^{\mu}$.

In double precision floating point arithmetic $\varepsilon_{\text {machine }} \approx 2.2204 e-016$. The following table indicates the values of constant $L_{\Gamma} \varepsilon_{\text {machine }}^{\mu}$ of the lower bound on $t o l$.

\begin{tabular}{|l|l|l|}
\hline curve & $\mathrm{n}=2$ & $\mathrm{n}=3$ \\
\hline Hilbert & $6.6640 e-008$ & $2.9666 e-005$ \\
\hline Schoenberg & $6.6042 e-004$ & $2.6310 e-001$ \\
\hline Mora & $6.9757 \mathrm{e}-013$ & $6.9757 \mathrm{e}-010$ \\
\hline Schoenberg- $\alpha$ & $1.3323 \mathrm{e}-012$ & $1.3323 \mathrm{e}-009$ \\
\hline Cherruault & $6.9757 \mathrm{e}-010$ & $6.9757 \mathrm{e}-007$ \\
\hline ADC1 & $4.4409 \mathrm{e}-013$ & $4.4409 \mathrm{e}-010$ \\
\hline ADC2 & $6.6613 \mathrm{e}-013$ & $6.6613 \mathrm{e}-010$ \\
\hline
\end{tabular}

Note that the $\alpha$-dense curve values are computed for $\sigma=1000$ and these lower bound constants are better than those of the space-filling curves. 
Another reasonable bound on the tolerance is the following. If there is a zero $x^{*}$ of $f$ and $x(t)$ is $\alpha$-dense, then there must be a point $t^{\prime}$ that $\left\|x(t)-x^{*}\right\| \leq \alpha$. Hence if $f \in \operatorname{Lip}_{L_{f}} \beta$, then

$$
\left\|f\left(r\left(t^{\prime}\right)\right)\right\| \leq L_{f}\left\|r\left(t^{\prime}\right)-x^{*}\right\| \leq L_{f} \alpha
$$

Hence $t o l \leq L_{f} \alpha$ seems to be a practical restriction because tol must be definitely less than $L_{f} \alpha$ to sort out the possible zero.

In comparison we give the density estimates of these curves as well $(\sigma=1000)$.

\begin{tabular}{|l|l|l|}
\hline curve & $\mathrm{n}=2$ & $\mathrm{n}=3$ \\
\hline Mora & 0.0033 & 0.0046 \\
\hline Schoenberg- $\alpha$ & 0.0061 & 0.0085 \\
\hline Cherruault & 0.0044 & 0.0054 \\
\hline ADC1 & 0.0022 & 0.0030 \\
\hline ADC2 & 0.0032 & 0.0044 \\
\hline
\end{tabular}

For the 2D test problems and $\sigma=1000$, the bound $L_{f} \alpha$ is proportional to 1e-2 except for the Powell problem No. 7 the Lipschitz constant of which is $1.7 \mathrm{e}+5$. In the case of $3 \mathrm{D}$ problems the smallest bounds are proportional also to 1e-2. Hence the selection of $t o l=1 e-2$ seems appropriate (for similar tolerance, see also Butz [10]).

8.2. Test results. We tested the four methods on each curve and on each $2 \mathrm{D}$ test problems with $t o l=1 e-2$ and $i$ tmax $=1 e+6$. The $\alpha$-dense curves used the parameter $\sigma=1000$. The test was carried out on a PC with Intel I7 processor and Matlab R2011b. A summary of the obtained results (average iteration/average precision) is contained in the following two tables, the first of which contains the best methods versus curves, while the second contains the best curves versus methods.

\begin{tabular}{|l||l|l|||l|l|}
\hline \multicolumn{1}{|c||}{} & \multicolumn{2}{c||}{ best } & in iterations & \multicolumn{2}{c|}{ best } & in precision \\
\hline 2D curve & method & iteration & method & precision \\
\hline Hilbert & $(\mathrm{d}-2)$ & $1.0848 \mathrm{e}+004$ & $(\mathrm{~d}-2)$ & $3.1479 \mathrm{e}-001$ \\
\hline Schoenberg & $(\mathrm{d}-1)$ & $1 \mathrm{e}+6$ & $(\mathrm{~d}-1)$ & $9.0372 \mathrm{e}-001$ \\
\hline Mora & $(\mathrm{d}-2)$ & $1.4541 \mathrm{e}+004$ & $(\mathrm{~d}-3)$ & $3.1661 \mathrm{e}-001$ \\
\hline Schoenberg- $\alpha$ & $(\mathrm{d}-2)$ & $1.2754 \mathrm{e}+005$ & $(\mathrm{~d}-1)$ & $3.0483 \mathrm{e}-001$ \\
\hline Cherruault- $\alpha$ & $(\mathrm{d}-2)$ & $4.7817 \mathrm{e}+004$ & $(\mathrm{~d}-3)$ & $1.9037 \mathrm{e}-001$ \\
\hline ADC1 & $(\mathrm{d}-2)$ & $1.1923 \mathrm{e}+004$ & $(\mathrm{~d}-4)$ & $1.9774 \mathrm{e}-001$ \\
\hline ADC2 & $(\mathrm{d}-2)$ & $1.6164 \mathrm{e}+004$ & $(\mathrm{~d}-4)$ & $3.1686 \mathrm{e}-001$ \\
\hline
\end{tabular}

\begin{tabular}{|c|c|c|c|c|}
\hline & best & in iterations & best & in precision \\
\hline method & curve & iteration & curve & precision \\
\hline$(d-1)$ & ADC2 & $3.7099 \mathrm{e}+004$ & Cherruault & $58 \mathrm{e}-001$ \\
\hline$(d-2)$ & Hilbert & $1.0848 \mathrm{e}+004$ & Cherruault & $1.9064 \mathrm{e}-001$ \\
\hline$(\mathrm{d}-3)$ & Hilbert & $1.4002 \mathrm{e}+004$ & Cherruault & $1.9037 \mathrm{e}-001$ \\
\hline$(d-4)$ & $\mathrm{ADC} 1$ & $4.5867 \mathrm{e}+004$ & Cherruault & $1.9065 \mathrm{e}-001$ \\
\hline
\end{tabular}


In fact, none of the methods solved test problem No. 7 and Schoenberg's space filling curve did perform poorly. On average, the other method-curve combinations performed acceptably. For 3D the situation became different. The next table contains the results (average iteration/average precision) of 3D test problems. Here we set itmax $=1 e+8$.

\begin{tabular}{|c|c|c|c|c|}
\hline & \multicolumn{2}{|c|}{ best in iterations } & \multicolumn{2}{|c|}{ best in precision } \\
\hline curve & method & iteration & method & precision \\
\hline Mora & $(\mathrm{d}-2)$ & $2.7581 \mathrm{e}+006$ & $(\mathrm{~d}-3)$ & $2.5842 \mathrm{e}+000$ \\
\hline Schoenberg- $\alpha$ & $(d-2)$ & $1.1053 \mathrm{e}+007$ & $(\mathrm{~d}-4)$ & $5.1233 \mathrm{e}-001$ \\
\hline Cherruault- $\alpha$ & $(\mathrm{d}-4)$ & $4.1809 \mathrm{e}+006$ & $(\mathrm{~d}-4)$ & $1.5270 \mathrm{e}-001$ \\
\hline ADC1 & $(d-2)$ & $2.0374 \mathrm{e}+006$ & $(d-1)$ & $2.6802 \mathrm{e}+000$ \\
\hline $\mathrm{ADC} 2$ & $(d-2)$ & $7.1008 \mathrm{e}+006$ & $(\mathrm{~d}-4)$ & $6.0737 \mathrm{e}-002$ \\
\hline
\end{tabular}

The performances of the 3D Hilbert and Schoenberg curves were so poor that the results are not included in the table. The 3D Hilbert curve performed better; however, the achieved precision was limited due to the lack of computer memory and a more efficient 3D Hilbert-curve program that computes the coordinate values directly. It is seen that $\alpha$-dense curves performed better and the best methods stopped under the exit condition $\left\|f\left(r\left(t_{i}\right)\right)\right\| \leq t o l$. The arguments of the previous subsection indicate that smaller tol would require smaller $\varepsilon_{\text {machine }}$, which can be obtained with multiple precision arithmetic.

In general, we can say that the proposed methods are working and feasible, although they are more expensive than the local methods. For higher dimension they require the use of multiple precision and more efficient curve computation other than those of 34, 35] and references cited therein.

\section{Appendix}

The test problems are taken from the Estonian test problem collection 36]. Whenever it is available we give the original source as well.

No. 1 (Yamamoto [37)

$$
\begin{aligned}
& f_{1}(x)=x_{1}^{2}+x_{2}^{3} \\
& f_{2}(x)=x_{2}^{2}
\end{aligned}
$$

No. 2 (Powell [38])

$$
\begin{aligned}
& f_{1}(x)=x_{1}-1 \\
& f_{2}(x)=x_{1} x_{2}-1 .
\end{aligned}
$$

No. 3 (Yamamoto 37)

$$
f_{1}(x)=x_{1}^{3}+x_{1} x_{2},
$$




$$
f_{2}(x)=x_{2}+x_{2}^{2} .
$$

No. 4 (Fuchs)

$$
\begin{aligned}
& f_{1}(x)=x_{1}^{2}-x_{2}^{2}-1, \\
& f_{2}(x)=x_{1}^{2}+x_{2}^{2}-4 .
\end{aligned}
$$

No. 5 (Brezinski 39])

$$
\begin{aligned}
& f_{1}(x)=0.5 x_{2}^{2}-0.5 \\
& f_{2}(x)=-x_{2}+\sin \left(x_{1}\right)+\sin \left(x_{2}-1\right)+1 .
\end{aligned}
$$

No. 6 (Bartish)

$$
\begin{aligned}
& f_{1}(x)=x_{1}^{2}+x_{2}^{2}-1, \\
& f_{2}(x)=0.75 x_{1}^{3}-x_{2} .
\end{aligned}
$$

No.7 (Powell [38])

$$
\begin{aligned}
& f_{1}(x)=10000 x_{1} x_{2}-1 \\
& f_{2}(x)=\exp \left(-x_{1}\right)+\exp \left(-x_{2}\right)-1.0001 .
\end{aligned}
$$

No. 8 (Boggs 40])

$$
\begin{aligned}
& f_{1}(x)=x_{1}^{2}-x_{2}+1, \\
& f_{2}(x)=x_{1}-\cos \left(0.5 \pi x_{2}\right) .
\end{aligned}
$$

No. 9 (Brezinski 39])

$$
\begin{aligned}
& f_{1}(x)=-x_{1}+0.5 x_{2}^{2}-1.5 \\
& f_{2}(x)=-x_{2}+0.605 \exp \left(1-x_{1}^{2}\right)+0.395
\end{aligned}
$$

No. 10 (Allgower-Georg [41])

$$
\begin{aligned}
& f_{1}(x)=\left(x_{1}-x_{2}^{2}\right)\left(x_{1}-\sin \left(x_{2}\right)\right), \\
& f_{2}(x)=\left(\cos \left(x_{2}\right)-x_{1}\right)\left(x_{2}-\cos \left(x_{1}\right)\right) .
\end{aligned}
$$

No.11 (Yamamoto 37)

$$
\begin{aligned}
& f_{1}(x)=x_{1}+x_{2}+x_{3}-1, \\
& f_{2}(x)=0.2 x_{1}^{3}+0.5 x_{2}^{2}-x_{3}+0.5 x_{3}^{2}+0.5, \\
& f_{3}(x)=x_{1}+x_{2}+0.5 x_{3}^{2}-0.5 .
\end{aligned}
$$

No. 12 (Allgower-Georg [41])

$$
\begin{aligned}
& f_{1}(x)=x_{1}^{2}+2 x_{2}^{2}-4 \\
& f_{2}(x)=x_{1}^{2}+x_{2}^{2}+x_{3}-8 \\
& f_{3}(x)=\left(x_{1}-1\right)^{2}+\left(2 x_{2}-\sqrt{2}\right)^{2}+\left(x_{3}-5\right)^{2}-4 .
\end{aligned}
$$


No. 13 (Brown-Conte)

$$
\begin{aligned}
& f_{1}(x)=3 x_{1}+x_{2}+2 x_{3}^{2}-3, \\
& f_{2}(x)=-3 x_{1}+5 x_{2}^{2}+2 x_{1} x_{3}-1, \\
& f_{3}(x)=25 x_{1} x_{2}+20 x_{3}+12 .
\end{aligned}
$$

No. 14 (Babitsch)

$$
\begin{aligned}
& f_{1}(x)=x_{1} x_{2}+x_{2} x_{3}+x_{1} x_{3}-47 \\
& f_{2}(x)=x_{1}^{1}+x_{2}^{2}-x_{3}^{2} \\
& f_{3}(x)=\left(x_{3}-x_{1}\right)\left(x_{3}-x_{2}\right)-2 .
\end{aligned}
$$

\section{REFERENCES}

1. Targonszky, G.: An always convergent iteration process. Acta Mathematica Hungarica, 4(1-2), (1953), 119-126.

2. Beauzamy, B.: On the paths to the zeros of a polynomial. J. of Inequal. Es Appl., 4, (1999), 83-89.

3. Sergeyev, Y. D. and Strongin, R. G.: Global Optimization with Non-Convex Constraints. Springer, 2000.

4. Zumbusch, G.: Parallel Multilevel Methods: Adaptive Mesh Refinement and Loadbalancing. B.G. Teubner, Stuttgart-Leipzig-Wiesbaden, 2003. ISBN: 3-519-00451-8.

5. Bebendorf, M.: Hierarchical Matrices. Springer, 2008.

6. Sergeyev, Y. D., Strongin, R. G., and Lera, D.: Introduction to Global Optimization Exploiting Space-Filling Curves. Springer, 2013.

7. Butz, A. R.: Space filling curves and mathematical programming. Information and Control, 12, (1968), 314-330.

8. Butz, A. R.: Convergence with Hilbert's space filling curve. Journal of Computer and System Sciences, 3, (1969), 128-146.

9. Strongin, R. G.: On the convergence of an algorithm for finding a global extremum. Engineering Cybernetics, 11(4), (1973), 549-555.

10. Butz, A. R.: Solutions of nonlinear equations with space filling curves. Journal of Mathematical Analysis and Applications, 37, (1972), 451-383.

11. Hlawka, E.: Über eine Klass von Näherungspolygonen zur Peanokurve. Journal of Number Theory, 43, (1993), 93-108.

12. Cherruault, Y.: Mathematical Modelling in Biomedicine. D. Reidel Publishing Company, Dordrecht, Holland, 1986.

13. Guillez, A.: Alienor, fractal algorithm for multivariable problems. Mathl Comput. Modelling, 14, (1990), 245-247.

14. Cherruault, Y. and Mora, G.: Optimisation Globale. Théorie des courbes $\alpha$-denses. Economica, Paris, 2005. ISBN 2-7178-5065-1.

15. Efimov, A. V.: Linear methods of approximating continuous periodic functions. Mat. Sb. (N.S.), 54(96), (1961), 51-90. (in Russian). 
16. Kolodil, I. M. And Khil'debrand, F.: Some properties of the modulus of continuity. Mat. Zametki, 9(5), (1971), 495-500. Also in Mathematical Notes, 9(5), (1971), 285-288.

17. Galántai, A. and Abaffy, J.: Always convergent iteration methods for nonlinear equations of Lipschitz functions. Numer Algor., 69, (2015), 443-453. DOI 10.1007/s11075014-9905-1.

18. AbAfFy, J. and Forgó, F.: Globally convergent algorithm for solving nonlinear equations. J. Optim. Theory App., 77, (1993), 291-304.

19. Pietrus, A.: A globally convergent method for solving nonlinear equations without the differentiability condition. Numerical Algorithms, 13, (1996), 60-76.

20. Singh, A. N.: The theory and construction of non-differentiable functions. Lucknow University Studies, Lucknow India, No. I.

21. Sagan, H.: Space-filling Curves. Springer, 1994.

22. BADER, M.: Space-Filling Curves An Introduction with Applications in Scientific Computing. Springer, 2013.

23. Butz, A. R.: Alternative algorithms for Hilbert's space-filling curve. IEEE Transactions on Computers, pp. 424-426.

24. Buckley, S.: Space-filling curves and related functions. Irish Mathematical Society Bulletin, 36, (1996), 9-18.

25. Bauman, K. E.: The dilation factor of the Peano-Hilbert curve. Mathematical Notes, 80(5), (2006), 609-620.

26. Jaffard, S. and Nicolay, S.: Pointwise smoothness of space-filling functions. Applied and Computational Harmonic Analysis, 26, (2009), 181-199.

27. Schoenberg, I. J.: On the Peano curve of Lebesgue. Bulletin of American Mathematical Society, 44, (1938), 519.

28. Schoenberg, I. J.: Mathematical Time Exposures. The Mathematical Association of America, 1994.

29. Steele, J. M.: Seedlings in the theory of shortest paths. in Grimmett, G. and Welsh, D. (eds): Disorder in Physical Systems: A volume in Honor of J. M. Hammersley. Cambridge University Press, London, 1990, pp. 277-306.

30. TAn, Y.: The construction of Lebesgue space-filling curve. Southeast Asian Bulletin of Mathematics, 36, (2012), 883-890.

31. Mora, G.: The Peano curves as limit of $\alpha$-dense curves. Rev. R. Acad. Cien. Serie A. Mat., 99(1), (2005), 23-28.

32. Rice, J. E.: Numerical Methods. McGraw-Hill, 1983.

33. Delahaye, J. P.: Sequence Transformations. Springer, 1988.

34. Breinholt, G. and Schierz, C.: Algorithm 781: Generating Hilbert's space-filling curve by recursion. ACM Transactions on Mathematical Software, 24(2), (1998), 184189.

35. Jin, G. and Mellor-Crumley, J.: SFCGen: A framework for efficient generation of multi-dimensional space-filling curves by recursion. ACM Transactions on Mathematical Software, 31(1), (2005), 120-148. 
36. Roose, A., Kulla, V., Lomp, M. and Meresso, T. (eds): Test Examples of Systems on Nonlinear Equations, Version 3-90. Estonian Software and Computer Service Company, Tallinn, 1990.

37. Үамамото, N.: Newton's method for singular problems and its applications to boundary value problems. J. Math. Tokushima Univ., 17, (1983), 27-88.

38. Powell, M. J. D.: A FORTRAN Subroutine for solving systems of nonlinear algebraic equations. in RABinowitz, P. (ed.): Numerical Methods for Nonlinear Algebraic Equations. Gordon and Breach Science Publisher, 1970, pp. 115-161.

39. Brezinski, C.: Algorithmes d'accélération de la convergence. Étude numérique. Editions Technip. Paris, 1978. ISBN 2-7108-0341-0.

40. Boggs, P. T.: The solution of nonlinear systems of equations by a-stable integration techniques. SIAM Journal on Numerical Analysis, 8(4), (1999), 767-785.

41. Allgower, E. L. and Georg, K.: Relationships between Deflation and Global Methods in the Problem of Approximating Additional Zeros of a System of Nonlinear Equations, in Eaves, B. C., Gould, F. J., Peitgen, H. O. and Todd, M. J. (eds): Homotopy Methods and Global Convergence. Plenum Press, New York, 1983. pp. 31-42. 